\section{La chimiokine CXCL12 et le papillomavirus humain}

\section{Une piste pour la pathogenèse du syndrome WHIM}

Emilie Brotin, Laetitia Carthagena, Ken Y.C. Chow, Françoise Bachelerie
દ. Brotin, L. Carthagena, F. Bachelerie :

Laboratoire cytokines, chimiokines

et immunopathologie, UMR-S996, Université Paris-Sud 11,

32, rue des Carnets, 92140 Clamart, France.

K.y.C. Chow : Leiden/Amsterdam Center for Drug Research,

Division of Medicinal Chemistry,

VU University Amsterdam, Amsterdam,

1081 HV, Pays-Bas.

francoise.bachelerie@u-psud.fr
Cet article est dédié à la mémoire de Dominique Emilie, directeur de l'UMR-S996

Le syndrome WHIM (SW) est un désordre immunohématologique rare caractérisé par une lymphopénie et une neutropénie et qui doit son acronyme aux manifestations cliniques des premiers cas décrits en 1990 (warts, hypogammaglobulinemia, bacterial infections, and myelokathexis) [1]. Quinze ans après, l'analyse génétique de cas familiaux a permis d'identifier que la majorité des patients étaient porteurs de mutations hétérozygotes du gène codant pour le récepteur CXCR4 [2]. Les cellules issues de ces patients présentent un gain de fonction de CXCR4 qui se manifeste par des réponses biologiques anormales et exacerbées à la chimiokine CXCL12, le ligand unique de CXCR4 [1]. Les bases moléculaires et le lien entre ces dysfonctions et les manifestations immunohématologiques du syndrome font l'objet d'un article publié dans ce numéro de médecine/sciences [11] $(\rightarrow)$.

Le propos de cette $(\rightarrow)$ Voir l'article de A. Bignon et al., page 391 de ce numéro nouvelle est la susceptibilité singulière des patients aux infections par les papillomavirus humains (HPV) dont on pense qu'elle a pour origine les dysfonctions de l'axe de signalisation formé par la paire primordiale CXCL12/CXCR4. Les membres de la famille des HPV présentent de grandes similitudes dans leur organisation génomique et leur cycle réplicatif
[3]. Ces virus infectent les épithéliums où ils effectuent leur cycle réplicatif de manière non lytique, et le contrôle de l'infection par les réponses immunitaires de l'hôte est limité et imprédictible. L'infection est souvent asymptomatique et peut se manifester par des lésions de la peau ou des muqueuses des sphères anogénitales ou orales qui, dans de rares cas associés à des virus à haut risque (par exemple HPV16 ou 18), peuvent évoluer en cancers [3]. En revanche, les patients souffrant du SW sont sujets à des infections chroniques et sévères par HPV qui se manifestent par une profusion de verrues et/ou des lésions des muqueuses qui progressent fréquemment en cancers [1], suggérant que les dysfonctionnments de l'axe CXCL12/CXCR4 pourraient contribuer à la pathogénie virale. S'exercent-elles directement comme un facteur de susceptibilité à l'infection et/ou indirectement via les désordres immunohématologiques du syndrome?

\section{De l'apport du WHIM à l'identification des relations entre HPV et la paire CXCL12/CXCR4}

La pathologie liée à l'infection par HPV constitue une manifestation majeure du SW qui affecte plus de $79 \%$ des patients et représente un facteur de risque de mortalité prématurée en raison du développement de cancers $[1,4,5]$. L'altération du nombre de cellules dendritiques myéloïdes et plasmacytoïdes circulantes observée chez ces patients pourrait s'étendre à la peau et contribuer à la pathogénie d'HPV [6]. Cependant, comment expliquer que les patients soient spécifiquement susceptibles aux infections par HPV? De plus, un récent essai vaccinal contre des sous-types d'HPV infectant les muqueuses démontre que les patients souffrant du SW ont la capacité de développer une réponse humorale et cellulaire anti-HPV [7]. Par ailleurs, les sérums de ces patients vaccinés sont capables de neutraliser l'infection par HPV in vitro, incitant à généraliser la vaccination préventive chez les jeunes patients atteints du SW [7]. Une première observation en faveur d'une contribution directe des dysfonctions de l'axe CXCL12/ CXCR4 à la pathogénie virale fut celle de la présence anormale de CXCL12 dans les kératinocytes des lésions de la peau et des muqueuses infectées par HPV chez des patients souffrant du SW. La chimiokine n'est en effet retrouvée ni dans l'épiderme sain ni dans d'autres pathologies de la peau, bénignes, inflammatoires ou virales [4]. Cependant, et de manière plus surprenante, nous avons aussi observé la présence anormale de la chimiokine dans les lésions HPV issues d'individus témoins ne souffrant pas du SW, suggérant un lien plus général entre les fonctions biologiques de CXCL12 et le cycle viral [4]. Ainsi dans la peau, CXCL12 produite par les fibroblastes exerce un effet mitogénique sur les kératinocytes [8] que le virus pourrait détourner au profit de sa propre réplication en modulant l'expression de la chimiokine dans 


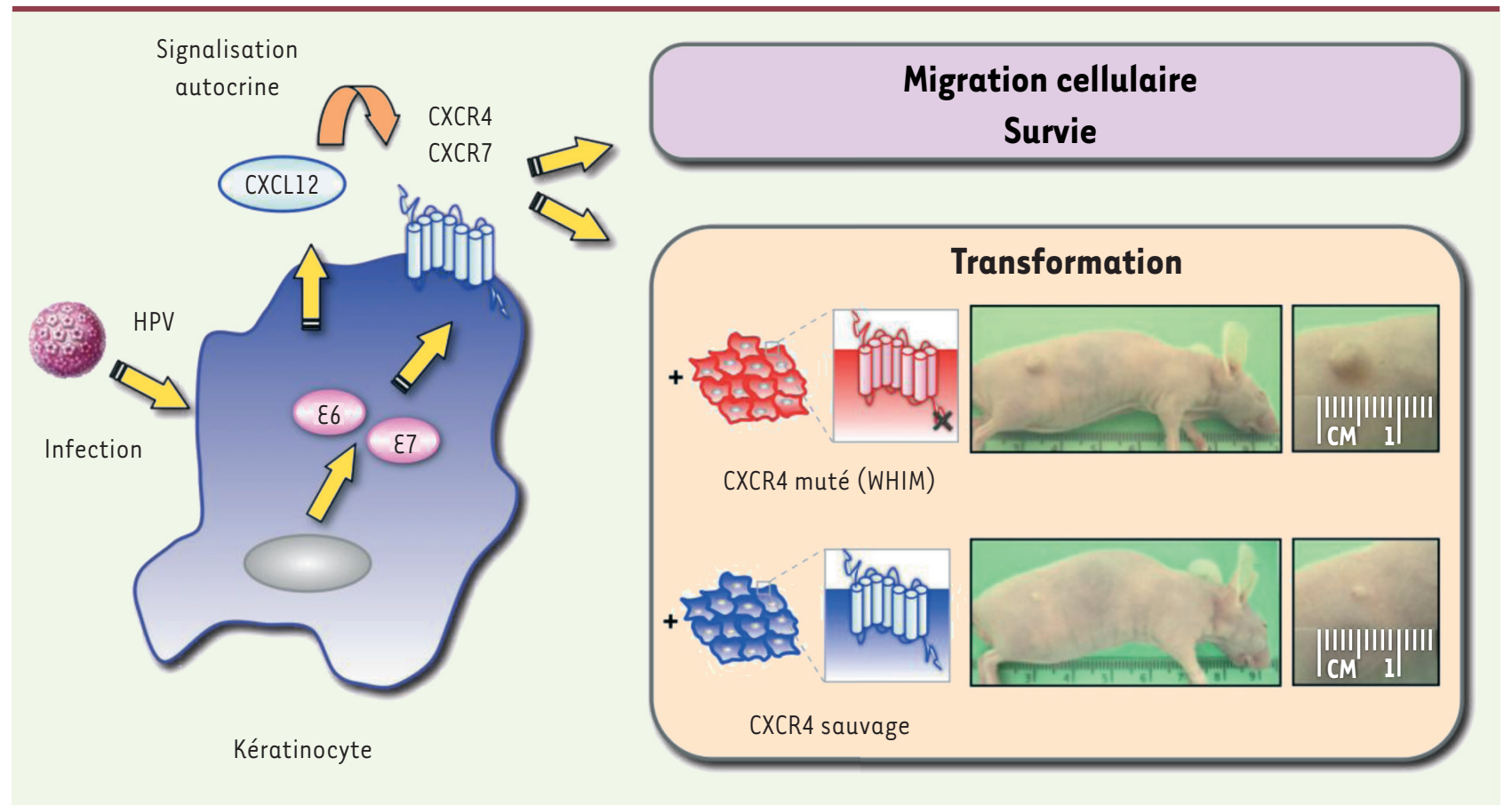

Figure 1. Relations entre HPV et l'axe de signalisation dépendant de CXCL12. Dans les kératinocytes immortalisés par HPV à haut risque, les oncoprotéines virales $\varepsilon 6$ et $\varepsilon 7$ induisent l'expression de CXCL12 et de ses récepteurs CXCR4 et CXCR7. Les interactions autocrines entre la chimiokine et ses récepteurs contrôlent la migration et la survie des kératinocytes. L'expression du récepteur CXCR4 mutant hyperactif associé au syndrome WHIM (CXCR41013) confère aux kératinocytes immortalisés un potentiel transformant.

ses cellules cibles. Dans le contexte du SW, cette balance pourrait être modifiée en faveur de la carcinogenèse associée à l'infection par HPV.

Impact fonctionnel des relations entre HPV et la paire CXCL12/CXCR4

Nous avons cherché à caractériser les liens observés entre CXCL12 et l'infection par HPV et, en premier lieu, nous avons déterminé s'ils pouvaient être reproduits in vitro. À l'instar des observations faites in vivo, les kératinocytes primaires n'expriment pas la chimiokine; en revanche, ils la produisent et la retiennent à leur surface lorsqu'ils sont immortalisés comme conséquence de l'expression de génomes HPV à haut risque [9]. Par extension, le niveau d'expression de CXCR4 à la surface de ces kératinocytes augmente, ainsi que celui de CXCR7, le second récepteur de CXCL12, suggérant que la chimiokine est engagée dans des interactions autocrines avec ses récepteurs [9].
L'utilisation d'antagonistes spécifiques de I'interaction CXCL12-récepteurs a effectivement permis de révéler le rôle critique de cet axe de signalisation dans la migration, la prolifération et la survie des kératinocytes immortalisés [9] (Figure 1). D’autre part, la réduction des niveaux des protéines virales $\varepsilon 6 / \varepsilon 7$, médiatrices dans l'immortalisation cellulaire [3], provoque de manière corollaire celle de CXCL12 et de ses récepteurs, indiquant que l'expression de ceux-ci est induite par les oncogènes viraux [9].

\section{Dysfonctionnement de CXCR4}

\section{et transformation cellulaire par HPV}

Sur la base de ces résultats et compte tenu de la fréquence des complications malignes des lésions induites par HPV chez les patients atteints du SW, l'hypothèse d'un rôle positif du gain de fonction de CXCR4 (par exemple la mutation CXCR4 ${ }^{1013}$ ) sur le potentiel oncogène d'HPV a été avancée. Afin d'évaluer cette possibilité, nous avons recherché si l'expression du mutant CXCR4 ${ }^{1013}$ dans les kératinocytes immortalisés pouvait conférer à ceux-ci le pouvoir transformant que la seule présence d'HPV ne suffit pas à enclencher. Des expériences in vivo de xénogreffes réalisées chez des souris nude ont révélé que les animaux auxquels ont été injecté des kératinocytes immortalisés exprimant le récepteur mutant CXCR4 ${ }^{1013}$ développaient des tumeurs solides. Ces observations, conjuguées à l'absence de nodules chez les animaux xénogreffés avec des kératinocytes immortalisés exprimant le récepteur CXCR4 sauvage [9] (Figure 1), suggèrent que les réponses biologiques anormales et exacerbées du récepteur mutant de la chimiokine CXCL12 favorisent la transformation cellulaire associée au virus.

\section{Conclusions et perspectives}

L'étude du SW s'est révélée particulièrement utile à l'identification du rôle inattendu que CXCL12 exerce dans 
l'infection HPV et la transformation qui lui est associée, et permet d'envisager des approches thérapeutiques basées sur le blocage des interactions de la chimiokine avec ses récepteurs. II reste à caractériser les mécanismes moléculaires qui contrôlent les liens fonctionnels entre l'axe CXCL12 et l'infection virale et qui concernent aussi bien les HPV à haut risque identifiés dans des lésions orales et génitales que ceux à bas risque, très rarement associés à des cancers, et retrouvés dans les verrues et les lésions anogénitales. La nature des HPV associés aux lésions malignes des patients souffrant du SW reste peu caractérisée. Dans les rares cas étudiés, des HPV à haut risque [5], mais aussi de manière surprenante des HPV à bas risque ont été détectés (nos observations non publiées). La sévérité de l'infection HPV associée au SW pourrait être la conséquence d'une exacerbation des liens entre l'axe CXCL12 et HPV due au gain de fonction de CXCR4. Ce mécanisme pourrait sous-tendre une plus grande permissivité des kératinocytes à l'infection. Étudier le cycle réplicatif d'HPV implique de recourir à des cultures épithéliales organotypi- ques qui constituent les seuls modèles de réplication virale in vitro car celleci a une stricte dépendance vis-à-vis de la différenciation des kératinocytes. Ces modèles restent difficiles à mettre en œuvre et sont particulièrement peu développés pour les virus à bas risque : comme ces virus sont incapables d'immortaliser les cellules, il est difficile d'obtenir des cultures de kératinocytes dans lesquelles le génome viral persiste. La description récente d'un processus d'immortalisation de kératinocytes primaires qui n'altère pas leurs caractéristiques [10] permet d'envisager une adaptation de ces modèles organotypiques à l'étude des HPV à bas risque. À terme ces travaux permettront de caractériser le nouveau rôle de la chimiokine CXCL12 et de ses récepteurs dans la pathogénie de l'infection par HPV. $\diamond$

WHIM syndrome: on the track of an interplay between human papillomavirus and the CXCL12 chemokine

\section{CONFLIT D'INTÉRÊTS}

Les auteurs déclarent n'avoir aucun conflit d'intérêts concernant les données publiées dans cet article.

\section{RÉFÉRENCES}

1. Kawai T, Malech HL. WHIM syndrome : congenital immune deficiency disease. Curr Opin Hematol 2009 ; $16: 20-6$.

2. Hernandez PA, Gorlin RJ, Lukens JN, et al. Mutations in the chemokine receptor gene CXCR4 are associated with WHIM syndrome, a combined immunodeficiency disease. Nat Genet 2003 ; 34 : 70-4.

3. Doorbar J. Molecular biology of human papillomavirus infection and cervical cancer. Clin Sci (Lond) 2006 ; $110: 525-41$

4. Balabanian K, Lagane B, Pablos JL, et al. WHIM syndromes with different genetic anomalies are accounted for by impaired CXCR4 desensitization to CXCL12. Blood $2005 ; 105: 2449-57$.

5. Cipriani NA, Blair $\varepsilon$, Taxy JB. WHIM syndrome and oral squamous cell carcinoma. Oral Surg Oral Med Oral Pathol Oral Radiol Endod 2010 ; 109 : 105-8.

6. Tassone L, Moratto D, Vermi W, et al. Defect of plasmacytoid dendritic cells in warts, hypogammaglobulinemia, infections, myelokathexis (WHIM) syndrome patients. Blood $2010 ; 116: 4870-3$

7. Handisurya A, Schellenbacher C, Reininger B, et al. A quadrivalent $\mathrm{HPV}$ vaccine induces humoral and cellular immune responses in WHIM immunodeficiency syndrome. Vaccine $2010 ; 28: 4837-41$.

8. Florin L, Maas-Szabowski N, Werner S, et al. Increased keratinocyte proliferation by JUN-dependent expression of PTN and SDF-1 in fibroblasts. J Cell Sci 2005 ; 118 : 1981-9.

9. Chow Ky, Brotin દ́, Ben Khalifa Y, et al. A pivotal role for CXCL12 signaling in HPV-mediated transformation of keratinocytes : clues to understanding HPVpathogenesis in WHIM syndrome. Cell Host Microbe $2010 ; 8: 523-33$.

10. Chapman S, Liu X, Meyers C, et al. Human keratinocytes are efficiently immortalized by a Rho kinase inhibitor. J Clin Invest 2010 ; 120 : 2619-26.

11. Bignon A, Biajoux V, Bouchet-Delbos L, et al. CXCR4, une cible thérapeutique dans certains déficits immunitaires rares? Med Sci (Paris) 2011 ; 27 : 391- 7.

\section{NOUVELLE}

\section{Une approche originale de sélection de nouveaux ARN non codants}

Mathieu Rederstorff

> Deux principales familles d'acides ribonucléiques ou ARN ont été identifiées jusqu'à présent dans tous les organismes vivants (voir Glossaire) [1] : les ARN codant pour des protéines ou ARN messagers, qui servent de matrice à la synthèse protéique, et les ARN non codants (ARNnc), qui ne sont pas traduits en protéines, mais dont la fonction est directement portée par I'ARN. De façon intéressante, l'analyse en haute résolution, réalisée par le consortium ENCODE (Encyclopedia of DNA elements), de près de $1 \%$ du génome humain, a révélé que $90 \%$ de ce dernier
AREMS, UHP-CNRS UMR 7214,

Faculté des sciences et technologies, Nancy Université, boulevard des Aiguillettes, BP 70239,

54506 Vandœuvre-lès-Nancy, France. mathieu.rederstorff@maem.uhp-nancy.fr

était transcrit [2], mais que seule une faible proportion des transcrits (1,5\%) était traduite en protéines. Ainsi, il a été suggéré qu'une part importante des transcrits restants $(88,5 \%)$ pouvaient constituer la source de nouveaux ARNnc régulateurs [11]. Certains auteurs prédisent même 